\title{
INSULIN INFUSION DURING CARDIAC SURGERY
}

\author{
Authors: A Shenderey MD, J Lam-McCulloch MSc, S Belo MD PhD FRCPC \\ Affiliations: Sunnybrook and Women's College Health Sciences Centre, University of \\ Toronto, 2075 Bayview Avenue, Room M3-200, Toronto, Ontario, Canada M4N 3M5.
}

INTRODUCTION: Hyperglycemia associated with insulin resistance is common during cardiac surgery even if patients are not diabetic. A prospective randomized study showed that intensive insulin therapy reduces mortality by $40 \%$ among critically ill patients (60\% after cardiac procedure). 'Use of postoperative insulin infusion in diabetic patients undergoing cardiac surgery significantly reduces major infectious morbidity. ${ }^{2}$ The appropriate management of intraoperative hyperglycemia remains controversial. The study was designed to retrospectively evaluate the effect of intraoperative insulin therapy to improve the results on glucose management in patients undergoing cardiac surgery.

METHODS : The medical records of all patients who underwent cardiac surgical procedures during the study period were reviewed retrospectively. Data was collected for an 18-month period when an intraoperative insulin infusion was administered to patients undergoing cardiopulmonary bypass $(\mathrm{CPB})$ at our institution (Insulin group, $\mathrm{n}=95$ ) and during a 6-month period before introduction of insulin infusion (no Insulin, group, $\mathrm{n}=58$ ). Insulin infusions ranged from 2-4 Units/hr prebypass to 4-8 during CPB.

RESULTS : The two groups were comparable with respect to all demographic data. Blood glucose was significantly lower in the Insulin group during surgery (Glucose CPB $\max 10.9 \pm 2.1 \mathrm{mmol} / \mathrm{L}$ vs $13.8 \pm 3.2 \mathrm{mmol} / \mathrm{L}, \mathrm{p}<0.001$; and Glucose CPB mean $10.1 \pm 1.9$ $\mathrm{mmol} / \mathrm{L}$ vs $12.3 \pm 2.6 \mathrm{mmol} / \mathrm{L}, \mathrm{p}<0.001)$. Mean blood glucose level upon intensive care unit arrival was significantly decreased in the Insulin group $(8.2 \pm 2.3 \mathrm{mmol} / \mathrm{L}$ vs $10.5 \pm 2.4 \mathrm{mmol} / \mathrm{L}, \mathrm{p}<0.001)$. No hypoglycemia was noted in the Insulin Group. Blood Potassium was significantly lower in the Insulin group during CPB (Potassium CPB mean $5.1 \pm 0.7 \mathrm{mEq} / \mathrm{L}$ vs $5.6 \pm 0.7 \mathrm{mEq} / \mathrm{L}, \mathrm{p}<0.001$; and Potassium $\mathrm{CPB}$ max $5.4 \pm 0.6$ $\mathrm{mEq} / \mathrm{L}$ vs $6.0 \pm 0.8 \mathrm{mEq} / \mathrm{L}, \mathrm{p}<0.001)$.

DISCUSSION: Insulin can be safely administrated to all patients during cardiac surgery without producing hypoglycemia. An insulin infusion was associated with lower blood glucose and potassium levels. Despite administration of insulin, normoglycemia was unattainable during cardiopulmonary bypass, which may be related to relative low insulin infusion. Additional studies are needed to determine a better protocol for optimal glucose control, and whether this affects outcome in patients undergoing open-heart surgery.

1. N Engl J Med 2001;345:1359-67. 
2. Ann Thorac Surg 1999;67:352-62. 